\title{
Classification of Botnet Attacks in IoT Smart Factory Using Honeypot Combined with Machine Learning
}

\author{
Seungjin Lee ${ }^{\text {Corresp., } 1}{ }^{,}$Azween Bin Abdullah ${ }^{1}$, Noor Zaman Jhanjhi ${ }^{1}$, Hoong Kok Sim ${ }^{1}$ \\ ${ }^{1}$ School of Computer Science \& Engineering, Taylor's University, No. 1 Jalan Taylor's, 47500, Subang Jaya, Selangor, Malaysia \\ Corresponding Author: Seungjin Lee \\ Email address: leeseungjin@sd.taylors.edu.my
}

Industrial revolution 4.0 with the breakthrough technological advances in 5G, and artificial intelligence has innovatively transformed the manufacturing industry from digitalization and automation to the new era of smart factories. A smart factory can do not only more than just produce products in a digital and automatic system, but also is able to optimize the production on its own by integrating production with process management, service distribution, and customized product requirement. A big challenge to the smart factory is to ensure that its network security can counteract with any cyber attacks such as botnet and DDos (Distributed Denial of Service), They are recognized to cause serious interruption in production, and consequently economic losses for company producers. Among many security solutions, botnet detection using honeypot has shown to be effective in some investigation studies. It is a method of detecting botnet attackers by intentionally creating a resource within the network with the purpose of closely monitoring and acquiring botnet attacking behaviors. For the first time, a proposed model of botnet detection was experimented by combing honeypot with machine learning to classify botnet attacks. A mimicking smart factory environment was created on loT device hardware configuration. Experimental results showed that the model performance gave a high accuracy of above $96 \%$, with very fast time taken of just 0.1 milliseconds and false positive rate at 0.24127 using random forest algorithm with Weka machine learning program. Hence, the honeypot combined machine learning model in this study was proved to be highly feasible to apply in the security network of smart factory to detect botnet attacks. 
2 Classification of Botnet Attacks in loT Smart Factory

3 Using Honeypot Combined with Machine Learning

4

5

6

7

8

\author{
Seungjin Lee ${ }^{1}$, Azween Abdullah ${ }^{1}$, NZ Jhanjhi. ${ }^{1}$, Kok Sim Hoong ${ }^{1}$ \\ ${ }^{1}$ School of Computer Science and Engineering, Taylor's University, No. 1 Jalan Taylor's, 47500, \\ Subang Jaya, Selangor, Malaysia
}

Corresponding Author:

Seungjin Lee ${ }^{1}$

School of Computer Science and Engineering, Taylor's University, No. 1 Jalan Taylor's, 47500, Subang Jaya, Selangor, Malaysia

Email address: leephael8707@gmail.com

\begin{abstract}
Industrial revolution 4.0 with the breakthrough technological advances in $5 \mathrm{G}$, and artificial intelligence has innovatively transformed the manufacturing industry from digitalization and automation to the new era of smart factories. A smart factory can do not only more than just produce products in a digital and automatic system, but also is able to optimize the production on its own by integrating production with process management, service distribution, and customized product requirement. A big challenge to the smart factory is to ensure that its network security can counteract with any cyber attacks such as botnet and DDos (Distributed Denial of Service), They are recognized to cause serious interruption in production, and consequently economic losses for company producers. Among many security solutions, botnet detection using honeypot has shown to be effective in some investigation studies. It is a method of detecting botnet attackers by intentionally creating a resource within the network with the purpose of closely monitoring and acquiring botnet attacking behaviors. For the first time, a proposed model of botnet detection was experimented by combing honeypot with machine learning to classify botnet attacks. A mimicking smart factory environment was created on IoT device hardware configuration. Experimental results showed that the model performance gave a high accuracy of above $96 \%$, with very fast time taken of just 0.1 milliseconds and false positive rate at 0.24127 using random forest algorithm with Weka machine learning program. Hence, the honeypot combined machine learning model in this study was proved to be highly feasible to apply in the security network of smart factory to detect botnet attacks.
\end{abstract}

\title{
Introduction
}


39

40

41

42

43

44

45

46

47

48

49

50

51

52

53

54

55

56

57

58

59

60

61

62

63

64

65

66

67

68

69

70

71

72

73

74

75

76

77

The Industrial Revolution 4.0 has brought a great innovation to the conventional manufacturing into the new era of smart factories [1]. The conventional factories involve automation or digitalization within each production process. This however make it very difficult to manage the entire production chain from general to specific levels. More innovatively, smart factory can effectively manage many processes in the production chain thanks to the use of many IoT (Internet of Things) devices. They are installed and interconnected with each other in every machine or equipment along the production chain. Hence a smart factory is advantageous in producing a variety of products according to customer's desire at better quality and higher productivity. And, IoT devices/equipment play a very important role in the operation and management of smart factories. A demand for the IoT equipment in smart factories has been increasing since 2012 as shown in Figure 1. Especially, in the last five years (2015-2020), the use of IoT devices has increased tremendously from 18.2 billion to 50 billion for application in the smart factories [2].

Additionally, as smart factories are combined with ICT (Information and Communications Technology), all the facilities and devices are connected at the central wireless communication. This allows data to be freely linked between the processes and provides a more systematic, integrated and optimal production environment. Efficiency in time management for production can be greatly enhanced with a minimal production cost. Therefore, products produced by smart factories become more competitive in the market.

Although IoT smart factories have been built and operated in the industry, standards of implementation for smart factories have yet to be established [4]. Basically, a smart factory consists of three aspects, i.e. interconnection, collaboration and execution, which all attribute to the manufacturing conceptualization of being adaptive and flexible [5]. This concept is reflected in the architecture of the smart factory operating on IoT system as shown in Figure 2 [10]. With four layers arranged hierarchically, it starts at the physical resource layer, followed by the networking layer and the application layer, and ends at the terminal layer. A manufacturing system in the smart factory can be assessed from different layers [6]. With the aim of transforming conventional factories into smart factories, in-depth research needs to look into all layers.

From the security perspective, research should focus more on the physical resource/sensing layer. As it is directly related to the vast usage of theIoT devices in order to reinforce the security network for smart factories. Finding any security-related issues is one of the priorities required for a smooth system operation by means of resolving any failover problems arising from the entire manufacturing chain [7]. Especially, the IoT devices are such as RFID (radio-frequency identification), CCTVs, PLC (programmable logic controller) equipment, sensor and main database servers are installed or located at the physical resource layer. Data transmission 
78 between these IoT devices can be easily affected in case that data leakage in smart factory

79

80

81

82

83

84

85

86

87

88

89

90

91

92

93

94

95

96

97

98

99

100

101

102

103

104

105

106

107

108

109

110

111

112

113

114

115

116

117 network occurs. In the worst case, data updates can be abused by unauthorized users [8].

To mitigate the impact of data leakage and data abuse, real-time detection of cyber attacks to smart factory obviously becomes an extremely important factor to take into consideration of developing and improving security network of the smart factory [9].

Network security in the smart factory is highly at risk of being under cyber attacks due to the interconnection of a huge number of IoT equipment. According to a recent report, instability is recognized as one of the biggest limitations out of 250 vulnerable features found in the IoT devices [3]. As a result, cyber attack to smart factories can easily spread to not only quality process control and production control, but also product design which can be analyzed or copied by unauthorized or illegal accesses. In the worse scenario, highly confidential information such as process know-how, requirements of data analysis, product design drawings, $R \& D$ results are shared outside of the smart factory. Such threats of information leakage can cause serious damages and economic losses to both manufacturing and business sectors. This kind of cyber attack can be done by the act of exploiting security vulnerabilities in the ICT system via remote control or surveillance of systems in the IoT smart factory.

One of the most serious cyber attacks to smart factory is botnet. An example of the botnet attack is a temporary unavailability on some commercial websites such as Amazon, Netflix, Twitter, CNN and PayPal. A notable case ever recorded is the attack on the Dyn DNS infrastructure, which mobilized 100,000 IoT devices (mainly CCTV cameras) in October 2016. Another example is the new Mirai-source-code being launched in 2017. These Mirai-induced IoT botnets have occurred frequently in the recent years with a very serious consequence. Therefore, it is very important and urgent to identify and mitigate IoT botnets through the development of new technologies for network security [11]. As the number of attacks has soared due to unstable IoT devices in the Internet infrastructure, smart factories undoubtedly are highly possible to become an ideal victim of the IoT botnet attack.

Among many detection methods, honeypot has been investigated to apply for detecting botnet attack in various studies in the recent years[12]. However, a huge volume of attacking data collected by honeypot is highly complex and non-classified. This causes to lower the efficiency of botnet detection by the honey method in term of time taken and accuracy. In order to improve the efficiency, it is crucial to focus on classifying botnet attacking information and obtaining botnet attacking behaviors (intrusion type in other words). In this particular area of dealing with big data, artificial intelligence or machine learning has recently been applied effectively to speed up data processing, and make prediction as well as detection [13]. Hence, it becomes very potential to apply machine learning for botnet classification, which is notably yet to be investigated in previous studies, especially in the smart factory environment.

Peer] Comput. Sci. reviewing PDF | (CS-2020:10:54089:1:1:NEW 2 Dec 2020) 
118

119

120

121

122

123

124

125

126

127

128

129

130

131

132

133

134

135

136

137

138

139

140

141

142

143

144

145

146

147

148

149

150

151

152

153

154

155

Therefore, this study was aimed to investigate the feasibility of combining honeypot with machine learning in developing a botnet detection model for IoT smart factories. In this work, a configuration of hardware representing a physical layout of a smart factory was built and installed with software of honeypot combined machine learning. The whole setup was then programmed to run for simulation to detect and classify botnet attacks into intrusion types.

\section{Problem statement}

The problem statement can be elaborated with following points,

- A huge amount of attacking data collected by honeypot is highly complex.

- Without data classification, efficiency of the honeypot model is low, since the current time taken is long but at low accuracy to detect botnet.

- A very limited number of studies focused on botnet detection for the smart factory.

- Strategy of using honeypot with machine learning has been suggested very recently with only study framework and lack of model verification for supporting.

Study approach

- Apply machine learning as a supporting tool for classifying botnet attacks captured in log files generated by honeypot. Selection of random forest algorithm for machine learning to improve the classification process. Model testing on a hardware configuration mimicking a real smart factory environment.

\section{Related work}

Various botnet detection methods and their rationale are described in the Taxonomy in Figure 3. Besides, a smart factory is layered into the perception (physical), communication, network, data and applications with the function of each, as shown in the following taxonomy of the same figure.

Comparison of various detection models are presented in Table 1. Real-time detection is a very important factor which smart factories seek for [14].

- Honeypot can respond to attacks in real time and attract attackers to deceptive assets instead of actual assets [15]. Whereas for binary, anomaly detection methods, the response to real-time is however slower than that of the honeypot method [16] [17].

- Binary detection is simple in the structure of using 1 and 0 . But its detection processing speed is too low to be compatible to smart manufacturing environment which seeks realtime detection [14] 
156

157

158

159

160

161

162

163

164

165

166

167

168

169

170

171

172

173

174

175

176

177

178

179

180

181

182

183

184

185

186

187

188

189

190

191

192

193

194

195

- Although the C\&C (command and control) method targets for HTTP-based botnet detection and expansion, its structure is not simple to implement and the detection result is high false-positive [18].

- In terms of cost effectiveness, honeypot has an advantage since it requires a relative low cost of construction and management [19].

With an increasing interest in the potential application of machine learning, it offers a new solution for detecting abnormalities in the malicious Internet traffic. In fact, the Internet traffic which allows communication between IoT devices is distinguished from the Internet connectivity which runs on a variety of web server Many Internet-connected devices are such as smartphones, computer, laptops using a variety of web servers. Moreover, for the IoT devices, patterns of the network traffic are repeated in the regularity of network ping with small packets for logging.

Applying machine learning in botnet detection for smart factories can become useful to enhance performance of the honeypot model in term of speeding up the processing time or detection time [21]. Interestingly, it is notified that very limited studies making attempts to mount both honeypot and machine learning on IoT device networks to target attack on the IoT traffic.

Table 2 summarizes a few studies in botnet detection using the approaches of honeypot and/or machine learning.

- IoT botnet detection is an approach used to design a detection model based on the binary when botnet attacks IoT device as a hypothesis [22]. Although monitoring algorithms for the infected IoT device are simple and easy through web services, capacity of the IoT devices has certain limits as a restriction in the IoT botnet detection.

- Another approach to detect botnet is using machine learning which gave a high accuracy in detection at $91.66 \%$ [23]. However one disadvantage of using machine learning approach is that fast detection is hard to achieve in the randomized number of packets. Consequently, the feasibility of applying this approach for smart manufacturing needs more research looking into real-time factor and accuracy.

- Botnet detection using honeypot integrated with IoT, named as IoT honeypot was studied in the environment of smart factories [24]. In comparison with the machine learning approach by Wang et al. (2020), the IoT honeypot approach is able to gather information at high speed with less resource consumption [5]. Although the IoT honeypot approach has been shown to be scalable by applying it to sandboxes IoT to support high protocols, more expansion in various situations and environments is needed with features to activate the architecture of the IoT devices [5].

Peer] Comput. Sci. reviewing PDF | (CS-2020:10:54089:1:1:NEW 2 Dec 2020) 
196

197

198

199

200

201

202

203

204

205

206

207

208

209

210

211

212

213

214

215

216

217

218

219

220

221

222

223

224

225

226

227

228

229

230

231

232

233

234

235

- One study suggested to apply machine learning to detect botnet in the smart factory environment [25]. On the one hand, the machine learning approach can reduce the cost as an advantage. On the other hand, low detection rate and high complexity and uncertainty are recognized as big limitations. Thus, it might not suitable for smart factories, unless a construction of machine learning with Kenta-aware intrusion tower system is built, which bears an additional cost.

- Advancing from the IoT honeypot approach and the machine learning approach, honeypot combined with machine learning named as honeypot machine learning uses learning logging for detection and tracking at high accuracy [26]. In accordance with most standard equipment at various functions, the honeypot machine learning approach is suitable for the performance of smart factory with a minimal resource required. Hence, it is likely to be adopted in the future [26].

Among those approaches being discussed, IoT botnet and honeypot machine learning approaches shows some effective results in detecting botnets. These two approaches are possible to trace through logging at low cost and are most cost-saving for the IoT devices. Notably, feasibility of applying the honeypot machine learning approach in the smart factory especially has yet to investigate in any studies so far as being highlighted in the recent review work [13]. Therefore, more research in botnet detection should look insight into this particular area for application in smart factory.

\section{Materials \& Methods}

\section{Proposed model}

This section is organized to focus on three main aspects. The first and second aspects mention configuration and simulation of hardware in a virtual smart factory environment. The last aspect presents algorithms of the honeypot detection model in combination with machine learning programming.

\section{Configuration of hardware for a virtual smart factory environment}

In the configuration setup, some IoT devices (camera, RFID, temperature sensors) and two raspberry pie devices ( $\mathrm{Pi} 1$ and $\mathrm{Pi}$ ) were used to create a virtual smart factory environment.

The first Raspberry Pi (Pi1) was assigned as the actual main IoT data collection server by installing Open CVS. It was responsible for transmitting collected IoT data to the main PC. T-pot platform was chosen because it was suitable for virtual experiments using raspberry and allowed to monitor real-time botnet detection through dashboards. The second raspberry pi (Pi2) was installed with a virtual server (VM) (T-pot platform) So that collection of detection information in such the environment was deliberately established. One assumption was that botnet attacked the raspberry pi 2 (honeypot server) using 10 feature botnet datasets. Raspberry Pi1 and Pi2 were 
236

237

238

239

240

241

242

243

244

245

246

247

248

249

250

251

252

253

254

255

256

257

258

259

260

261

262

263

264

265

266

267

268

269

270

271

272

273

274

installed on a log server to keep the information on IoT product line in the factory and records of botnet attack pattern time zone, making it easy to track.

Operating mechanism of the honeypot combined machine learning model in smart factory is illustrated in Figure 4. When an attacker attempted to inject a malicious code through an open port. This step was done by logging into an IoT device at the physical resource/perception layer by combining multiple IDs and passwords. The honeypot intentionally broke into his protective wall and came in as a person who could reach the attacker. The main intention was to obtain information about attackers and malicious code botnets by recording each activity between the device and the intruder in the form of a log file. These log files captured information that allowed administrators to identify characteristics, transformations, target device types, $\mathrm{C} \& \mathrm{C}$ server IP addresses, port numbers of the new malware suites or botnets. Log file data was then converted to an appropriate table format that can be used as a dataset.

A machine learning (ML) tool was then trained with the dataset which contained 10 network parameters. These parameters were based on the most 10 common features of IoT botnet attacks to smart factory reported in the previous work studying in network architecture and network type used in the physical layer of smart factory, smart home network and smart city [27] [28] [29]. Algorithm written for this ML tool classified botnet data using R-studio and Weka. Memoryefficient classification was desirable to predict useful information by using less training data to prevent IoT devices from becoming overwhelming. Afterwards, appropriate measures were taken according to the results of the classification. Whenever the course exceeded the allowed size of training data, it would dynamically repeat.

\section{Simulation of hardware design with raspberry Pi transfer log files}

Each product in the smart factory was attached with a RFID (Radio-frequency identification) tag containing information. Camera reads the RFID tags to collect product information. The collected information was then stored in the raspberry Pi1 and Pi2 as the calling terminals. In other words, it was programmed to transmit the collected data to the IoT service server of the network through a terminal (camera, temperature, and RFID - IoT devices). The information kept in Pi1 and Pi2 as a log file was then transferred to the server of the main PC. This process simulation is illustrated in Figure 5.

\section{Raspberry Pi1 setting - Data transporting Open CV}

Transporting and receiving of data in this virtual environment werer similar to those taking place in the real smart factory. Data transporting Open CV was used as a collection of Python classes that transferred Open CV images and data from the raspberry Pi1 to the main computer via Data transporting Open CV messages.

Peer] Comput. Sci. reviewing PDF | (CS-2020:10:54089:1:1:NEW 2 Dec 2020) 
275 For example, on the main computer screen, video and picture streams were shown

276 simultaneously by sending signal data of raspberry Pil as shown in Figure 6. Algorithm was

277 required for the main PC and raspberry Pil for such data transfer as shown in Table 3.

278

279 Raspberry Pi2 setting - T-pot honeypot platform

280 After Raspberry Pi1 setting with data transporting Open CV, Raspberry Pi2 was set with T-pot

281 honeypot platform followed by virtual machine (VM). Verification and testing were done on all

282 the honeypot to be balanced at runtime. To do this task, a studio state command line was used to

283 write script and verify the transmission of log file.

284

285 The script in Figure 7 shows the load on the platform, the status of each honeypot, and the 286 uptime. Furthermore, the data collected by the honeypot was visually displayed using the Kibana 287 dashboard showing network attacked by malicious users and botnet. The Kibana dashboard 288 shown in Figure 8 is convenient and comprehensive for analysis of the type, location, and 289 malicious threat actors of botnet attacks. It infiltrated the Raspberry Pi server inside the VM. 290 This had many potential uses for data systems and metrif collection in smart manufacturing 291 environment that required real-time monitoring.

292

293

294

295

296

297

298

299

300

301

302

303

304

305

306

307

308

309

310

311

312

313

\section{Honeypot and machine learning classification process design and algorithms}

Diagram in Figure 9 describes the process design for botnet detection by honeypot combined with machine learning classification model. The entire process consisted of two stages. In the first stage (honeypot simulation), it took place at the raspberry Pi2 server which finished its loading by checking botnet credentials and then started the honeypot detection in the T-pot platform.

To verify botnet credentials in this step, a user name and password must be provided. Accurate information given by authorized users would be proceeded to start honeypot detection. The verification step took approximately 60 seconds.

Moving to stage 2 - log data collect, data which was earlier obtained from the authorized users in stage 1 , was being processed through a series of steps, i.e. retrieve logs, extract records, filter, text processing, upload, end automated process. During the text processing step before uploading, this was where machine learning was integrated with the honeypot for the purpose of classifying botnet attacks as illustrated in Figure 9.

As a result of classification, botnet attacking behaviors or botnet intrusion types were obtained and therefore used for machine learning training to detect botnets. After machine learning classification, processed data was converted to output result file and uploaded to end the 314 
315 Instruction to verify the codes and dataset:

316

317

318

319

320

321

322

323

324

325

326

327

328

329

330

331

332

333

334

335

336

337

338

339

340

341

342

343

344

345

346

347

348 The calculation of indices was performed using the following equation.

349

350 can balance the dataset for four categories of botnet attacks).

2. Make setting in the computer for dataset with frame set and honeypot log file.

4. Proceed to filter, extract records, review logs and test processing. each classified category. (DDos, Dos, Reconnaissance and Theft).

7. Call in the algorithm (random forest or SVM) to start classification. positive rate, and p-value.

\section{Results} other study.

\section{Dataset} and physical layer in smart factories, we experimented with data set 10 features of this paper[30]., which is the most suitable data set for this study. the botnet intrusion types. Details of the 10 features are presented in Table 5, which was evaluated for their statistical measurement values.

$$
x_{i}=\left(x_{i}-x_{\min }\right) * \frac{(b-a)}{\left(x_{\max }-x_{\min }\right)}+a
$$

1. Read the package setting for CARET, DPLYR, and READR in the library (this package

3. Start the test based on the 10 best feature data and wait until the true setting to come out.

5. Apply (flgs_number, srate, drate, rate, max, state_number, mean, min, stddev, seq) to

6. Obtain log files as samples for machine learning classifying into four types of botnets

8. Predict botnets based on the classification result in term of accuracy, time taken, false

In this section, results were collected mainly from the machine learning classification of botnet attacks. The data collection for the experiment was randomized if the reference was used. The experiment was carried out using Raspberry Pi and a personal computer. Features of the selected dataset were first described in Dataset section. To perform the classification on the dataset, honeypot combined machine learning model simulation was run by two techniques i.e. Weka, and Rstudio. The collected results were evaluated by comparison between the two, and with

In order to use a machine learning method to identify botnet as the target of IoT-based network

Dataset used for classification was selected based on ten features which were closely related to extracted from a direct comparison of entropy and correlation scores in the previous study [31] [30]. Specifically, transmission formation was calculated as correlation indices which were 
351

352

353

354

355

356

357

358

359

360

361

362

363

364

365

366

367

368

369

370

371

372

373

374

375

376

377

378

379

380

381

382

383

384

385

386

387

388

389

390

Model simulation was evaluated using some of the evaluation metrics of machine learning as shown in Table 6 [30]. Based on the results, the model can be evaluated whether it is highly efficient in optimization and able to reduce the error of drate. The values of max and min were to represent the values of training and response. Examples of correlation are in Figure 10 [30]..

\section{Classification by Weka-machine learning}

Classification result of the Weka-machine learning technique for four types of botnet attacks namely DDoS, DoS, reconnaissance, and theft is shown in Figure 11. For 76 instances, the average percentage of correct prediction (accuracy) for all four botnet attacks achieved $96.00526 \%$. The kappa statistic showed the model stability was 0.9466 with a mean absolute error of just 0.0478 . In term of accuracy and precision for each type of attacks, reconnaissance has the highest values.

Furthermore, after collecting pcap files from the virtual settings, statistical measures using correlation coefficients and entropy techniques were adopted to extract flow data using Argus tools in order to evaluate datasets based on the 10 best features. A new function was created based on the transaction flow of network connections to find out normal and intrusive events. A machine learning model has been trained and validated in different versions of datasets to assess the value of datasets compared to other datasets as shown in Figure 12.

\section{Classification by Rstudio-machine learning}

Figure 13 shows the results of classification using Rstudio machine learning technique. Two methods were used, i.e. support-vector machine (SVM) and random forest (RF). RF was calculated using the decision tree (DT) to predict mean values. RF was selected because it showed the effective detection in discrete datasets such as botnet [32]. Evaluation of the classification result were based on nine parameters namely, sensitivity, specificity, pos pred value, neg prered value, prevalence, detection rate, detection prevalence, balance accuracy, average.

In the RF method, a high accuracy was achieved at 0.96 . The $96 \%$ CIs were 0.8875 and 0.9917 . The RF provides Kappa at 0.964 . However, for SVM method, the accuracy was obtained at 0.7733 which is much lower than that of the RF. The $96 \%$ CIs were $0.6666,0.8621$. As can be seen in Figure 13, classification of DDoS attack type is highly efficient using Rstudio with respect to all 9 evaluating parameters. The detection rate and detection prevalence have low probabilities of 0.24 and 0.25 respectively. The overall detection using R-studio showed a statistically significant result since $\mathrm{p}$-value is less than $5 \%$.

\section{Discussion}

The two machine learning programs (Weka and Rstudio) following the random forest algorithm showed good result of classification and comparable with another study as shown in Figure 14. 
391 In the study of Mathur et al., botnet were detected via mining of the network traffic flow with 392 random committee method [33]. The resultant accuracy of the random committee was achieved 393 at $95.3 \%$, which was $1.3 \%$ lower than those obtained in this study at $96.66667 \%$ for random 394 forest-Weka and $96 \%$ for random forest-Rstudio. In term of time taken, both random forest395 Weka and random committee were able to detect botnets within a very short time of 0.00001 396 seconds or 0.1 milliseconds. Whereas it took a very much longer time of 19.37 second for the

397 398

399

400

401

402

403

404

405

406

407

408

409

410

411

412

413

414

415

416

417

418

419

420

421

422

423

424

425

426

427

428

429 random forest-Rstudio to detect botnets because of its program package. In addition, the p-value representing the significance of the detection models, $\mathrm{p}$ value to the random forest-Rstudio was less than $5 \%(2.2 \times 10-16)$, which showed that the detection model is statistically significant. As mentioned earlier that real time detection is the key factor importance to the network security of the smart factory, random forest method is therefore considered to be highly suitable for the environment of smart factory operating $24 / 7$ in time.

The result of this study can be said to be just relatively comparable with the work of Mathur et al. (2018), since both of the studies were based on network traffic flow and targeting at the botnet attack [33]. However, for the feasibility to apply in the smart factory environment, this study shows to be more feasible because of two reasons. First, the experiments were conducted on simulation of hardware specifically configured to mimic the real smart factory environment using IoT devices as mentioned in the proposed model section, which is lacked in the work of Mathur et al. (2018). Secondly, the experimental results obtained in this study addressed directly to the three deciding factors (time taken - 0.1 millisecond, accuracy - above 96\% and FPR 0.24127 , refer to Table 7 ) which are very useful for evaluating any tested methods being applied in the smart factory.

Whereas the latest publication work in 2020 reported that their study was based on smart factory ambient/environment to detect context-aware intrusion using machine learning [34]. But there was no mention in that study to target any specific types of cyber attacks or virus, but only for anomaly signs. Another limitation of the study is that its result just stated a very general possibility of process improvement of 29\% from 1.29\% [34]. Without showing the three deciding factors (time taken, accuracy and FPR), it can be hardly possible to evaluate the feasibility to apply for the smart factory. In addition to this point, the part of using machine learning used for training to obtain intrusions did not mention to include the best features of smart factory in the datasets. If including it would be very helpful to increase the feasibility or applicability of any detection models at the physical layers with interconnection of many IoT devices, as this present study were conducted accordingly. Furthermore, the Kibana platform supporting for a visualization of the system/model performance could provide a user-friendly interface for the administrators in the smart factory to analyze from a variety of perspectives more than just a visible display. 
430 This study had provided a basic background for developing a security network just for the smart 431 factory environment with a mimicking IoT device hardware configuration and random

432 algorithms for the experimental work. The results can be used as reference points or benchmarks 433 for more comparison with other future studies relating to smart factory. In fact, the number of 434 studies focusing on the security network for smart factory to target specifically botnet attacks 435 using honeypot is currently scarce throughout the literature, future work can based on this smart 436 factory hardware configuration design for the experimental testing for models or systems. Also, 437 it is suggested to further this study by conducting experiments in a real smart factory. By doing 438 that, a better result can be obtained for analysis when many factors of smart factories are taken 439 into consideration. Instead, a controlled virtual smart factory environment was created in this 440 study. The expected results will be more valuable for improving the productivity of smart 441 factories.

442

\section{Conclusions}

444 In this work, the model of combining honeypot with machine learning was proved to be feasible

445

446

447

448

449

450

451

452

453

454

455

456

457

458

459

460

461

462

463

464

465

466

467

468

in detecting botnet in the smart factory. Since the botnet can be easily spread into IoT smart factory environment with a high risk, hardware-based simulation and classification using random forest algorithm for Weka machine learning program showed a very good result. 96.66667 \% for accuracy, 0.1 milliseconds were achieved for the proposed model to detect botnet and the FPR was low at just 0.24127 . Comparing this result to other studies showed that the proposed model (honeypot combined machine learning to detect and classify botnet attack) in the smart factory was evaluated to be better because of three outstanding advantages. First, IoT devices were used in the hardware simulation configured to mimic the real smart factory environment. Second, the result of model testing has showed that with a short time taken - 0.1 millisecond,high accuracy above $96 \%$ and low FPR -0.24127 by the random forest Weka machine learning as the deciding factors. Lastly, machine learning have been used the dataset which included the best 10 features of the smart factory for training to obtain intrusions.

\section{Acknowledgements}

The author acknowledge the support of Taylor's University, School of Computer Science and Engineer in carrying out this experimental research work. This research work has been underwent for English proofreading prior to submitting to this journal.

\section{References}

[1] E. Oztemel and S. Gursev, "Literature review of Industry 4.0 and related technologies," J. Intell. Manuf., vol. 31, no. 1, pp. 127-182, 2020.

[2] M. S. Smith, "Protecting Privacy in an IoT-Connected World.," Inf. Manag. J., vol. 49, no. 6, pp. 36-39, 2015. 
469 [3] E. Casalinuovo, "Thematic Investment Opportunity - Internet of Things," Trop. Comment.,

470

471

472

473

474

475

476

477

478

479

480

481

482

483

484

485

486

487

488

489

490

491

492

493

494

495

496

497

498

499

500

501

502

503

504

505

506

507

508

509 vol. 4, no. 1, pp. 1-4, 2019.

[4] D. Guo, R. Y. Zhong, S. Ling, Y. Rong, and G. Q. Huang, "A roadmap for Assembly 4.0: self-configuration of fixed-position assembly islands under Graduation Intelligent Manufacturing System,” Int. J. Prod. Res., vol. 58, no. 15, pp. 4631-4646, 2020.

[5] Jiafu, Wan,Shenglong, Tang,Zhaogang, Shu,Di, Li,Shiyong, Wang, Muhammad, Imran,Athanasios, V. Vasilakos., "Software-Defined Industrial Internet of Things in the Context of Industry 4.0,” IEEE Sens. J., vol. 16, no. 20, pp. 7373-7380, 2016.

[6] X. Li, D. Li, J. Wan, C. Liu, and M. Imran, "Adaptive transmission optimization in SDNbased industrial internet of things with edge computing," IEEE Internet Things J., vol. 5, no. 3, pp. 1351-1360, 2018.

[7] S. Mittal, M. A. Khan, D. Romero, and T. Wuest, "Smart manufacturing: Characteristics, technologies and enabling factors," Proc. Inst. Mech. Eng. Part B J. Eng. Manuf., vol. 233, no. 5, pp. 1342-1361, 2019.

[8] K. S. H. Ramos, M. A. S. Monge, and J. M. Vidal, "Benchmark-based reference model for evaluating botnet detection tools driven by traffic-flow analytics," Sensors (Switzerland), vol. 20, no. 16, pp. 1-31, 2020.

[9] Brett, Stone-Gross, Marco, Cova, Lorenzo, Cavallaro, Bob, Gilbert, Martin, Szydlowski, Richard, Kemmerer, Christopher, Kruegel, Giovanni, Vigna, "Your botnet is my botnet: Analysis of a botnet takeover," in Proceedings of the ACM Conference on Computer and Communications Security, 2009, pp. 635-647.

[10] B. Chen, J. Wan, L. Shu, P. Li, M. Mukherjee, and B. Yin, "Smart Factory of Industry 4.0: Key Technologies, Application Case, and Challenges," IEEE Access, vol. 6, no. c, pp. 6505-6519, 2017.

[11] M. Ozcelik, N. Chalabianloo, and G. Gur, "Software-Defined Edge Defense Against IoTBased DDoS,” IEEE CIT 2017 - 17th IEEE Int. Conf. Comput. Inf. Technol., pp. 308-313, 2017.

[12] F. Ja' fari, S. Mostafavi, K. Mizanian, and E. Jafari, “An intelligent botnet blocking approach in software defined networks using honeypots," J. Ambient Intell. Humaniz. Comput., no. $0123456789,2020$.

[13] L. Seungjin, A. Abdullah, and N. Z. Jhanjhi, "A review on honeypot-based botnet detection models for smart factory," Int. J. Adv. Comput. Sci. Appl., vol. 11, no. 6, pp. 418-435, 2020 .

[14] G. Katz, P. Piantanida, and M. Debbah, "Distributed Binary Detection with Lossy Data Compression," IEEE Trans. Inf. Theory, vol. 63, no. 8, pp. 5207-5227, 2017.

[15] P. Duessel, C. Gehl, U. Flegel, S. Dietrich, and M. Meier, "Detecting zero-day attacks using context-aware anomaly detection at the application-layer,” Int. J. Inf. Secur., vol. 16, no. 5, pp. 475-490, 2017.

[16] F. Gerstmayer, J. Hausladen, M. Kramer, and M. Horauer, "Binary protection framework for embedded systems,” 2017 12th IEEE Int. Symp. Ind. Embed. Syst. SIES 2017 - Proc., 2017. 
510 [17] F. Fenzl, R. Rieke, Y. Chevalier, A. Dominik, and I. Kotenko, "Continuous fields: Enhanced

511

512

513

514

515

516

517

518

519

520

521

522

523

524

525

526

527

528

529

530

531

532

533

534

535

536

537

538

539

540

541

542

543

544

545

546

547

548 in-vehicle anomaly detection using machine learning models," Simul. Model. Pract. Theory, vol. 105, no. June, p. 102143, 2020.

[18] G. Fedynyshyn, M. C. Chuah, and G. Tan, "Detection and classification of different botnet C\&C channels," Lect. Notes Comput. Sci. (including Subser. Lect. Notes Artif. Intell. Lect. Notes Bioinformatics), vol. 6906 LNCS, pp. 228-242, 2011.

[19] A. Aziz, "A soft-decision fusion approach for multiple-sensor distributed binary detection systems,” IEEE Trans. Aerosp. Electron. Syst., vol. 47, no. 3, pp. 2208-2216, 2011.

[20] W. Zhang, B. Zhang, Y. Zhou, H. He, and Z. Ding, "An IoT Honeynet based on Multi-port Honeypots for Capturing IoT attacks,” IEEE Internet Things J., vol. PP, no. c, p. 1, 2019.

[21] M. Lim, A. Abdullah, N. Jhanjhi, M. Khurram Khan, and M. Supramaniam, "Link prediction in time-evolving criminal network with deep reinforcement learning technique," IEEE Access, vol. 7, pp. 184797-184807, 2019.

[22] S. K. Choi, C. H. Yang, and J. Kwak, "System hardening and security monitoring for IoT devices to mitigate IoT security vulnerabilities and threats," KSII Trans. Internet Inf. Syst., vol. 12, no. 2, pp. 906-918, 2018.

[23] W. Wang, Y. Shang, Y. He, Y. Li, and J. Liu, "BotMark: Automated botnet detection with hybrid analysis of flow-based and graph-based traffic behaviors," Inf. Sci. (Ny)., vol. 511, pp. 284-296, 2020.

[24] S. Dowling, M. Schukat, and H. Melvin, "A ZigBee honeypot to assess IoT cyberattack behaviour," 2017 28th Irish Signals Syst. Conf. IEEE, pp. 1-6, 2017.

[25] S. T. Park, G. Li, and J. C. Hong, "A study on smart factory-based ambient intelligence context-aware intrusion detection system using machine learning," Journal of Ambient Intelligence and Humanized Computing, vol. 0, no. 0, Springer Berlin Heidelberg, p. 0, 2018.

[26] R. Vishwakarma, "A Honeypot with Machine Learning based Detection Framework for defending IoT based Botnet DDoS Attacks," 2019 3rd Int. Conf. Trends Electron. Informatics, no. Icoei, pp. 1019-1024, 2019.

[27] Y. Fan et al., "SNPL: One scheme of securing nodes in iot perception layer," Sensors (Switzerland), vol. 20, no. 4, pp. 1-21, 2020.

[28] Z. A. Almusaylim and N. Zaman, "A review on smart home present state and challenges: linked to context-awareness internet of things (IoT)," Wirel. Networks, vol. 25, no. 6, pp. 3193-3204, 2019.

[29] M. Humayun, N. Z. Jhanjhi, M. Z. Alamri, and A. Khan, "Smart Cities and Digital Governance," Employing Recent Technol. Improv. Digit. Gov. IGI Glob., pp. 87-106, 2019.

[30] N. Koroniotis, N. Moustafa, E. Sitnikova, and B. Turnbull, "Towards the development of realistic botnet dataset in the Internet of Things for network forensic analytics: Bot-IoT dataset," Futur. Gener. Comput. Syst., vol. 100, pp. 779-796, 2019. 
549 [31] Y. Zheng and C. Keong, A feature subset selection method based on high-dimensional mutual information, vol. 13, no. 4. 2011.

551

552

[32] S. H. Kok, A. Abdullah, and N. Z. Jhanjhi, "Early detection of crypto-ransomware using pre-encryption detection algorithm,” J. King Saud Univ. - Comput. Inf. Sci., 2020.

553

[33] L. Mathur, M. Raheja, and P. Ahlawat, "Botnet Detection via mining of network traffic 554 flow," Procedia Comput. Sci., vol. 132, pp. 1668-1677, 2018.

555

[34] S. T. Park, G. Li, and J. C. Hong, "A study on smart factory-based ambient intelligence context-aware intrusion detection system using machine learning," J. Ambient Intell. Humaniz. Comput., vol. 11, no. 4, pp. 1405-1412, 2020. 


\section{Table $\mathbf{1}$ (on next page)}

Comparison of honeypot with other detection methods 
1

Table 1. Comparison of honeypot with other detection methods.

\begin{tabular}{|c|c|c|c|c|}
\hline & Honeypot & Binary detection & Anomaly detection & $\begin{array}{l}\text { C\&C } \\
\text { detection }\end{array}$ \\
\hline Configuration & $\begin{array}{l}\text { High-interaction } \\
\text { virtual server }\end{array}$ & Binary & $\mathrm{P} 2 \mathrm{P}$ & $\begin{array}{l}\text { Command \& } \\
\text { control server }\end{array}$ \\
\hline Advantages & $\begin{array}{l}\text { Monitor the } \\
\text { interaction of the grid } \\
\text { with infected devices } \\
\text { User friendly UI } \\
\text { system }\end{array}$ & $\begin{array}{l}\text { Easily applicable } \\
\text { to multi- } \\
\text { connection } \\
\text { systems }\end{array}$ & $\begin{array}{l}\text { Systems have the } \\
\text { capability to detect } \\
\text { zero-day attacks as } \\
\text { well }\end{array}$ & $\begin{array}{l}\text { Able to detect } \\
\text { and expand } \\
\text { HTTP-based } \\
\text { botnets. }\end{array}$ \\
\hline \multirow[t]{2}{*}{ Disadvantages } & $\begin{array}{l}\text { The analysis of } \\
\text { information on an } \\
\text { attack is slow and } \\
\text { passive. }\end{array}$ & $\begin{array}{l}\text { Spend a lot of } \\
\text { time training }\end{array}$ & $\begin{array}{l}\text { Not simple structure } \\
\text { high false-positive }\end{array}$ & $\begin{array}{l}\text { Not simple } \\
\text { structure } \\
\text { High false- } \\
\text { positive }\end{array}$ \\
\hline & {$[20][15]$} & {$[16]$} & [17] & {$[18]$} \\
\hline
\end{tabular}

2

3 


\section{Table 2 (on next page)}

A comparison of studies in botnet detection using honeypot and/or machine learning approaches for smart factories. 
1 Table 2. A comparison of studies in botnet detection using honeypot and/or machine learning

2 approaches for smart factories.

\begin{tabular}{|c|c|c|c|c|}
\hline Approaches & Strengths & Weaknesses & Research gap & Ref. \\
\hline $\begin{array}{l}\text { Machine } \\
\text { learning for } \\
\text { smart } \\
\text { factory } \\
\text { environment }\end{array}$ & Cost reduction & $\begin{array}{l}\text { Detection rate is } \\
\text { very low and the } \\
\text { accuracy is low. } \\
\text { The system is } \\
\text { complicated. }\end{array}$ & $\begin{array}{l}\text { Building machine learning } \\
\text { and Kenta-aware intrusion } \\
\text { tower systems for } \\
\text { information that will be } \\
\text { leaked from manufacturing } \\
\text { processes }\end{array}$ & [25] \\
\hline
\end{tabular}

\begin{tabular}{|c|c|c|c|c|}
\hline IoT Botnet & $\begin{array}{l}\text { Monitoring } \\
\text { Web-based } \\
\text { real-time IoT } \\
\text { equipment. } \\
\text { Easy and } \\
\text { simple } \\
\text { interface }\end{array}$ & Limited capacity & $\begin{array}{l}\text { New optimization requires } \\
\text { expansion of utilization }\end{array}$ & [22] \\
\hline $\begin{array}{l}\text { Machine } \\
\text { learning }\end{array}$ & $\begin{array}{l}91.66 \% \text { graph- } \\
\text { based detection } \\
\text { accuracy }\end{array}$ & $\begin{array}{c}\text { Difficult to } \\
\text { apply flow- } \\
\text { based detectors }\end{array}$ & $\begin{array}{l}\text { A graph-based bot mark is } \\
\text { required to increase the } \\
\text { accuracy of botnet detection }\end{array}$ & [23] \\
\hline $\begin{array}{l}\text { IoT } \\
\text { Honeypot }\end{array}$ & $\begin{array}{l}\text { Speed of } \\
\text { gathering } \\
\text { information is } \\
\text { fast. } \\
\text { Less resource } \\
\text { consumption. }\end{array}$ & $\begin{array}{l}\text { Unnecessary } \\
\text { data piles up }\end{array}$ & $\begin{array}{l}\text { It is necessary to activate } \\
\text { network protocol by } \\
\text { expanding IoT equipment } \\
\text { and sandbox. }\end{array}$ & {$[5]$} \\
\hline $\begin{array}{l}\text { Honeypot } \\
\text { machine } \\
\text { learning }\end{array}$ & $\begin{array}{l}\text { Real-time } \\
\text { monitoring } \\
\text { with the } \\
\text { combination of } \\
\text { honeypot and } \\
\text { machine } \\
\text { learning }\end{array}$ & $\begin{array}{l}\text { It is greatly } \\
\text { affected by the } \\
\text { system } \\
\text { environment. }\end{array}$ & $\begin{array}{l}\text { Problems with device data } \\
\text { capacity cloud server } \\
\text { application. }\end{array}$ & [26] \\
\hline
\end{tabular}




\section{Table 3(on next page)}

Algorithm of data transfer in raspberry Pi 1. 
1 Table 3. Algorithm of data transfer in raspberry Pi 1.

2

\begin{tabular}{|c|c|}
\hline \multicolumn{2}{|c|}{ Algorithm: Raspberry Pi image data transfer } \\
\hline 1. Input. & t: List of data transfer \\
\hline 2. $\quad(-1$ & Output: PI image data (task mapped to VM) \\
\hline 3. & Begin \\
\hline 4. & True: $C V, V M$. Show streamed images and data \\
\hline 5. & If \\
\hline 6. & two tasks get data from RFID, image, signal then \\
\hline 7. & Pick task with earliest \\
\hline 8. & Else \\
\hline 9. & Transfer data \\
\hline 10. & while VM \\
\hline 11. & Compute Utilization \\
\hline 12. & Sent image, signal each \\
\hline 13. & Repeat If data is available \& task allocated then \\
\hline 14. & Migrate task to less utilized data \\
\hline 15. & Else \\
\hline 16. & Start scheduling \\
\hline 17. & Until all send images as stream \\
\hline 18. & Image $=$ Pi Cam read \\
\hline 19. End & \\
\hline
\end{tabular}

3 


\section{Table 4(on next page)}

Algorithm for data classification. 
1 Table 1. Algorithm for data classification.

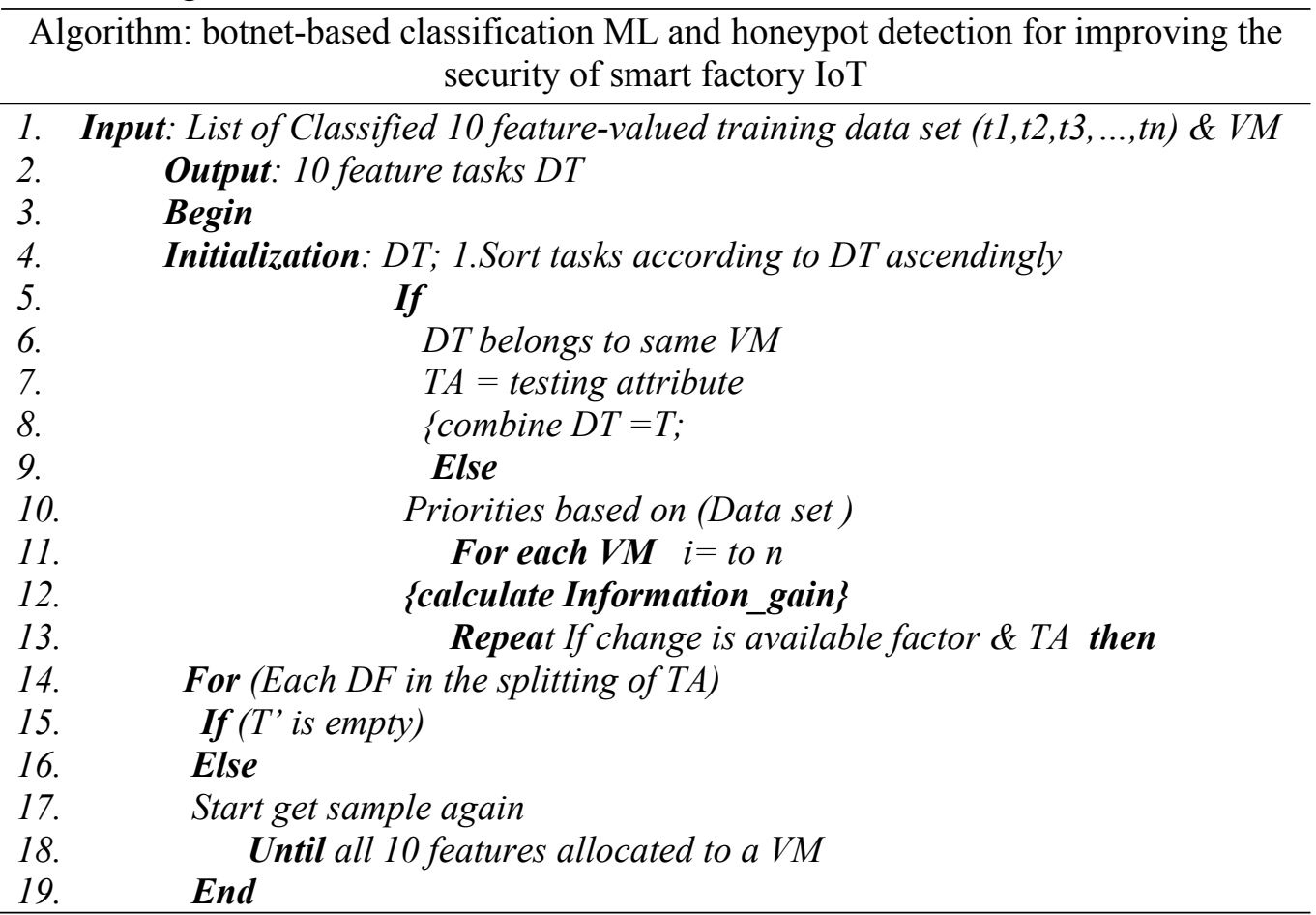




\section{Table 5 (on next page)}

10 Features of the selected dataset for classification. 
1 Table 1. 10 Features of the selected dataset for classification.

\begin{tabular}{ccl}
\hline No. & Name of features & \multicolumn{1}{c}{ Description } \\
\hline 1 & srate & Foundation-to-target t time packets for each second \\
2 & drate & Target-to-foundation packets for each second \\
3 & rate & Over-all packets for each second in transaction \\
4 & max & Maximum period of collected archives Source \\
5 & state_number & Numerical illustration of characteristic state \\
6 & mean & Average period of collecting records \\
7 & min & Minimum time of collecting records \\
8 & stddev & Standard deviation of aggregated records Total \\
9 & flgs_number & Numerical representation of feature flags \\
10 & seq & Argus sequence number \\
\hline
\end{tabular}

2

3

4 


\section{Table 6(on next page)}

Machine learning evaluation metrics. 
1 Table 6. Machine learning evaluation metrics.

\begin{tabular}{cl}
\hline Accuracy & ACC $=\frac{\mathrm{TP}}{T P+F P}$ \\
Precision & $P P V=\frac{T P}{\mathrm{TP}+F P}$ \\
Recall & $T P R=\frac{\mathrm{TP}}{\mathrm{TP}+\mathrm{FN}}$ \\
Fall-out & $F P T=\frac{F P}{F P+T N}$ \\
\hline
\end{tabular}

Note:

TP (True Positive): number of botnet containers represent as botnet FP (False Positive): number of regular containers symbolized as botnet TN (True Negative): number of normal containers represent as standard traffic FN (False Negative): number of botnet containers symbolized as standard traffic

2

3 


\section{Table 7 (on next page)}

Result comparison between random forest with random committee algorithms 
1 Table 7. Result comparison between random forest with random committee algorithms.

\begin{tabular}{lccccc}
\hline & Accuracy & $\begin{array}{c}\text { Time taken } \\
\text { (detection } \\
\text { time) }\end{array}$ & $\begin{array}{c}\text { Fall positive } \\
\text { rate (FPR) }\end{array}$ & p-value & Ref. \\
\hline $\begin{array}{l}\text { Random forest- } \\
\begin{array}{l}\text { Weka } \\
\text { Random forest- }\end{array}\end{array}$ & $\begin{array}{c}96.66667 \\
\%\end{array}$ & $0.0001 \mathrm{~s}$ & 0.24127 & - & $\begin{array}{c}\text { This } \\
\text { study }\end{array}$ \\
Rstudio & $96 \%$ & $19.37 \mathrm{~s}$ & 0.219 & 0.05 & - \\
\hline Random committee & $95.3 \%$ & $0.0001 \mathrm{~s}$ & 0.219 & - & {$[33]$} \\
\hline
\end{tabular}

2

3

4 


\section{Figure 1}

\section{Demand for loT equipment.}

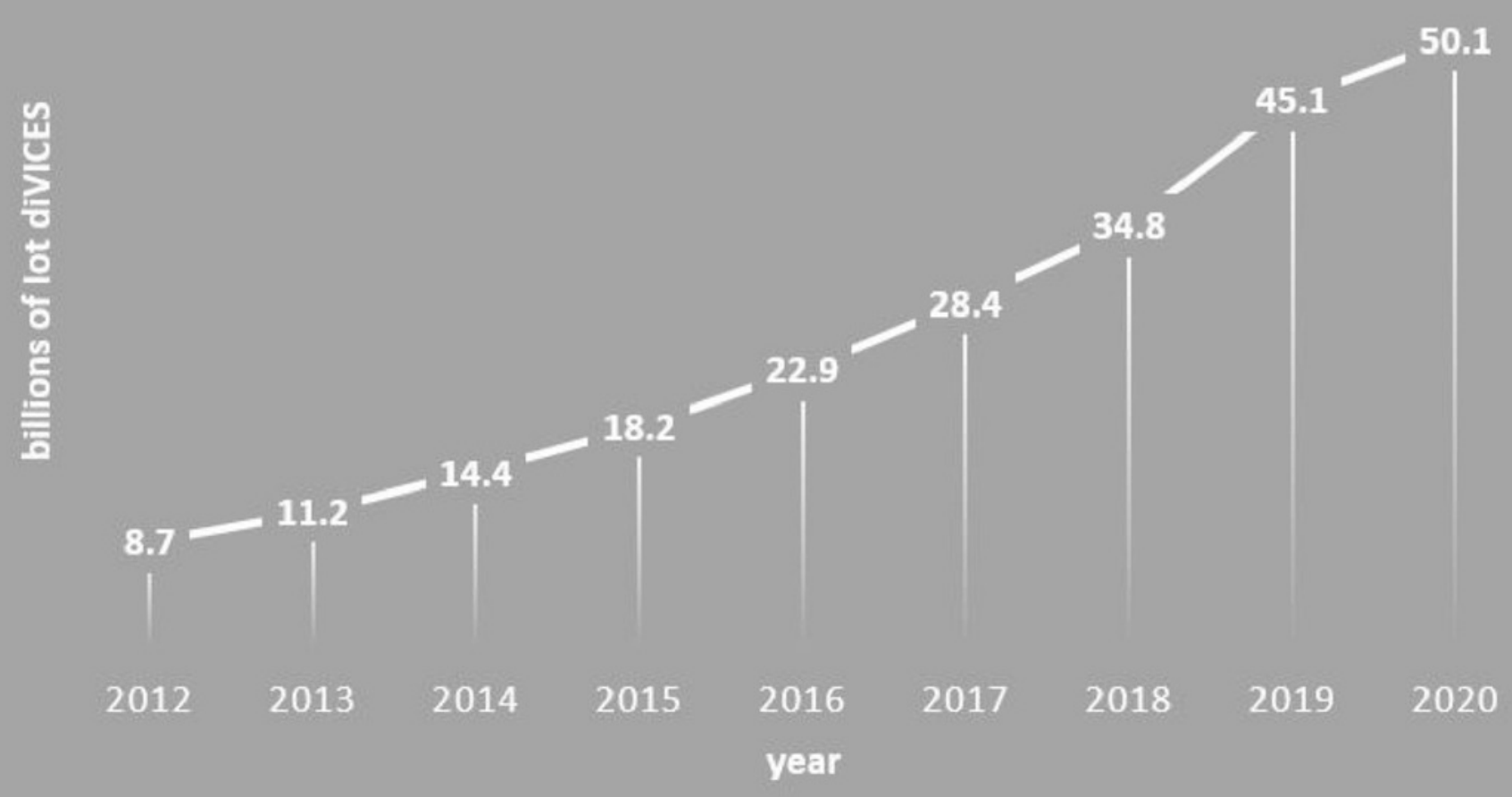


Figure 2

\section{Function requirements of smart factory.}
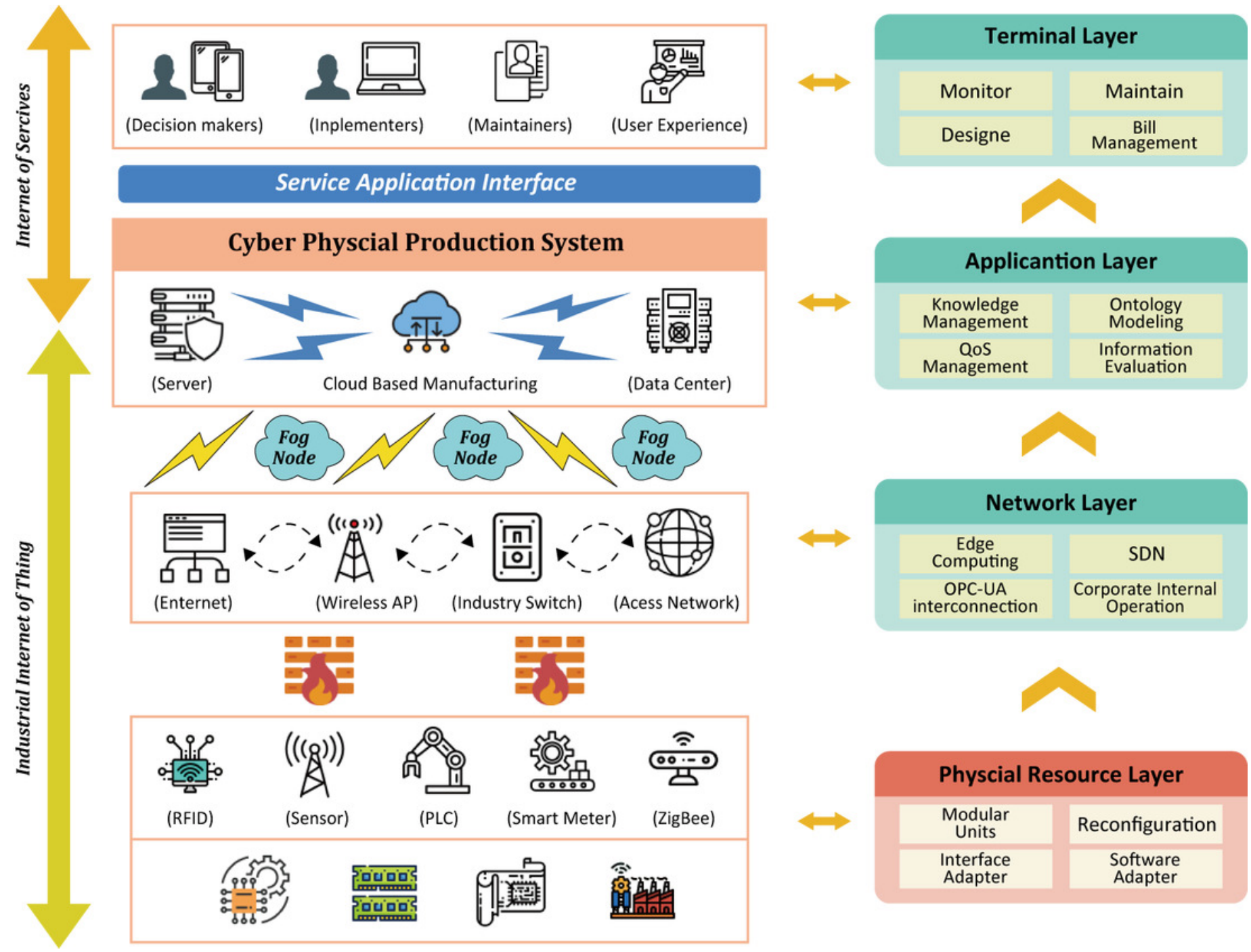

\section{Applicantion Layer}

\begin{tabular}{c|c} 
Knowledge & Ontology \\
Management & Modeling \\
QoS & $\begin{array}{c}\text { Information } \\
\text { Evaluation }\end{array}$
\end{tabular}

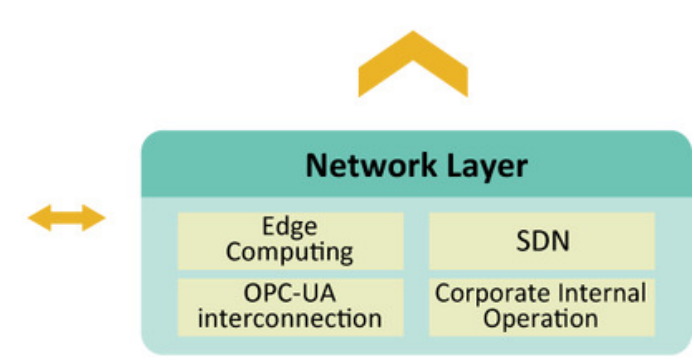


Figure 3

Taxonomies for botnet detection and security layers of IoT smart factories.
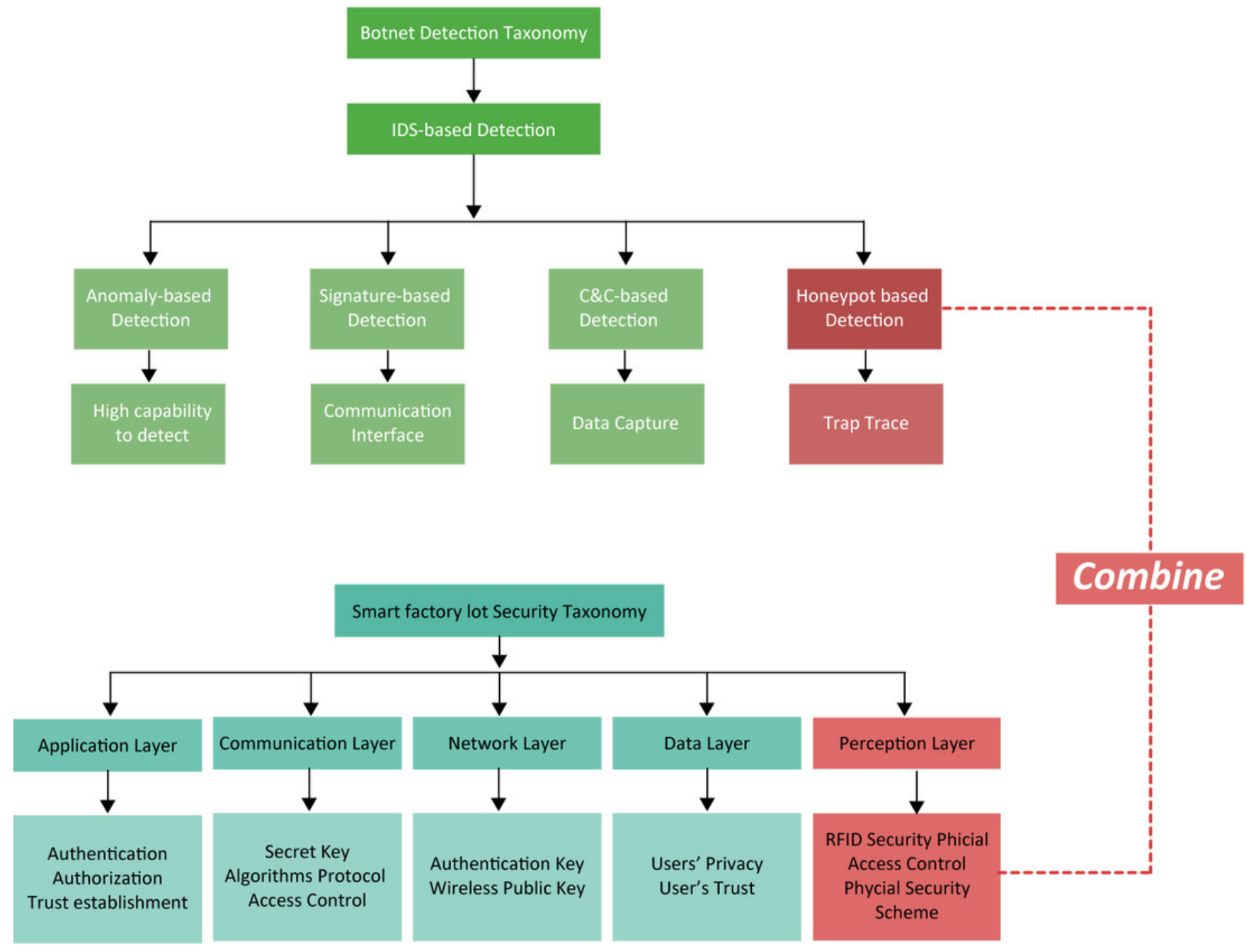
Figure 4

Operating mechanisms of botnet attacks to the physical sensing layer of a smart factory and design of the honeypot combined machine learning model.

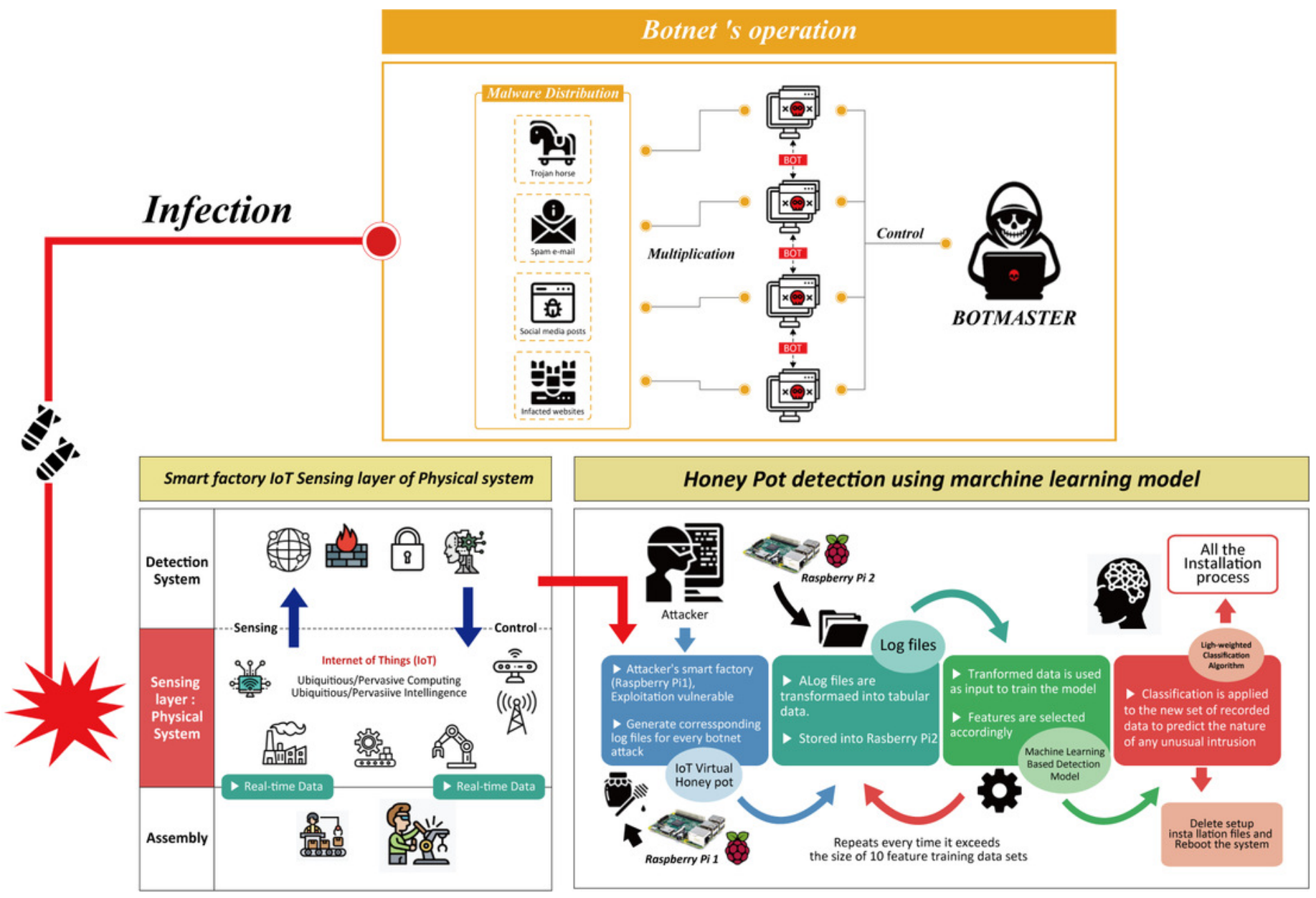




\title{
Figure 5
}

\author{
Simulation of hardware design
}

\section{HONEY-POT BASED BOTNET DETECTION FOR IoT in SMART FACTORIES}

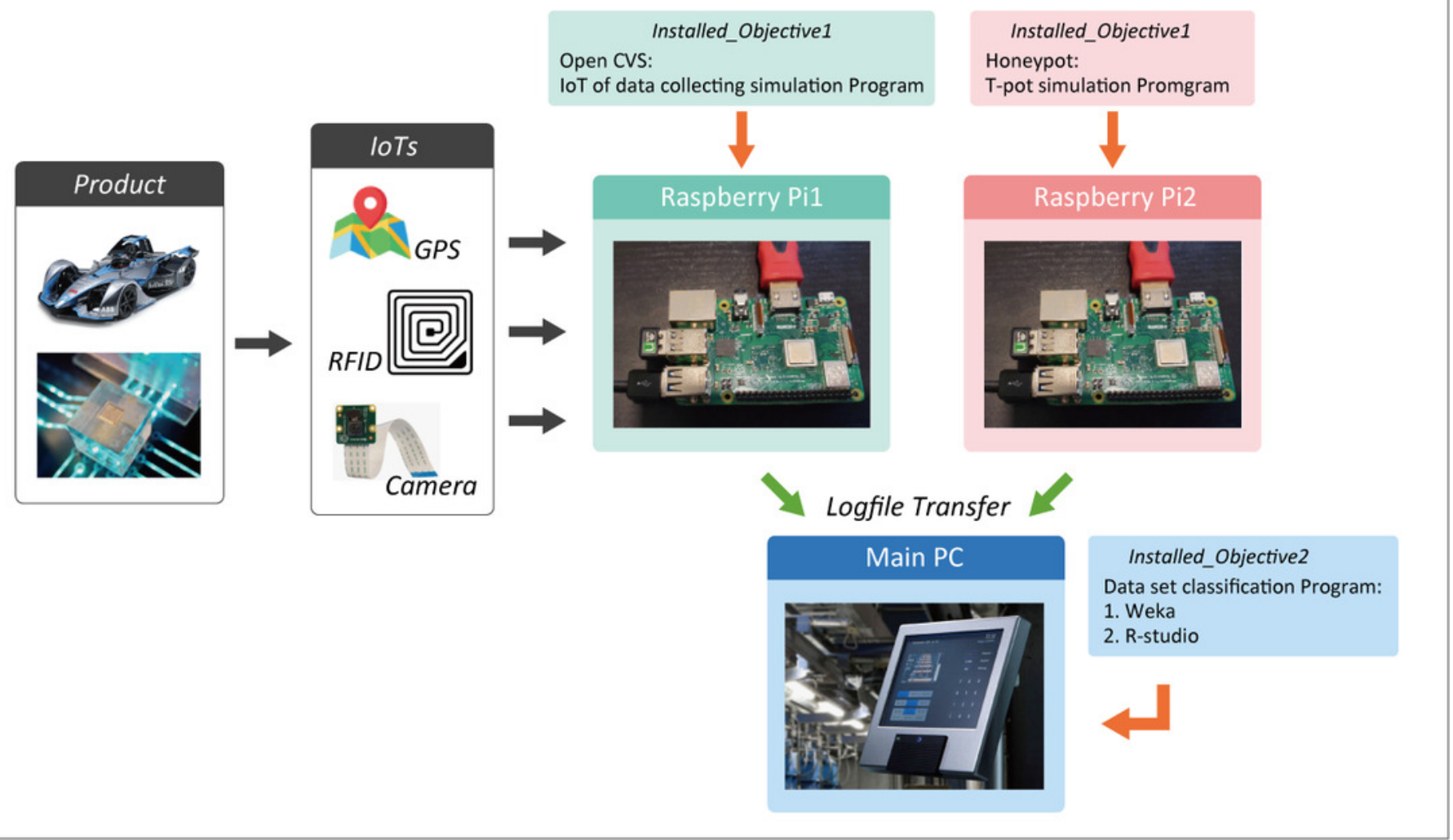




\section{Figure 6}

Pi Image data image.

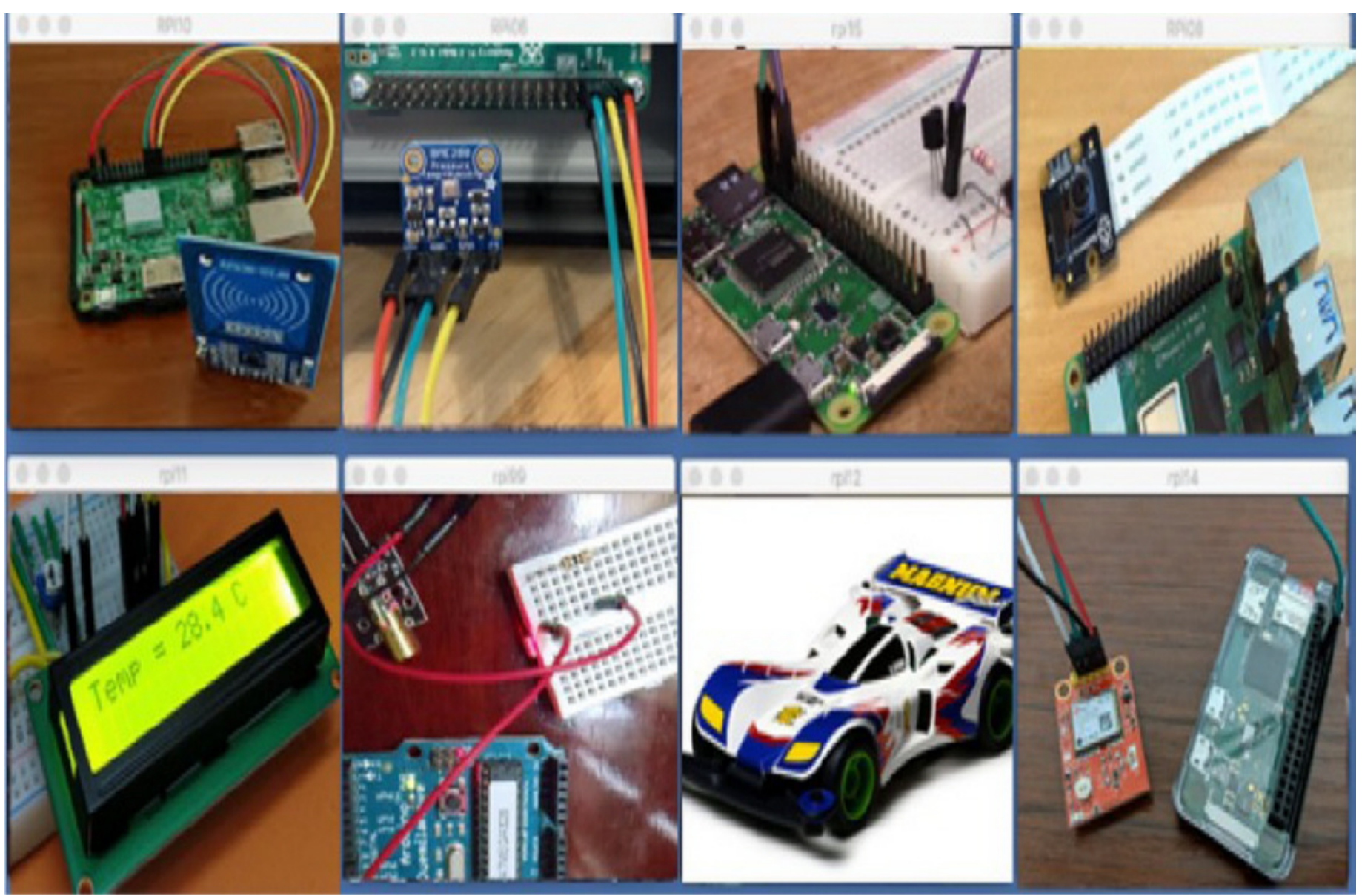


Figure 7

T-pot test script for raspberry Pi2.

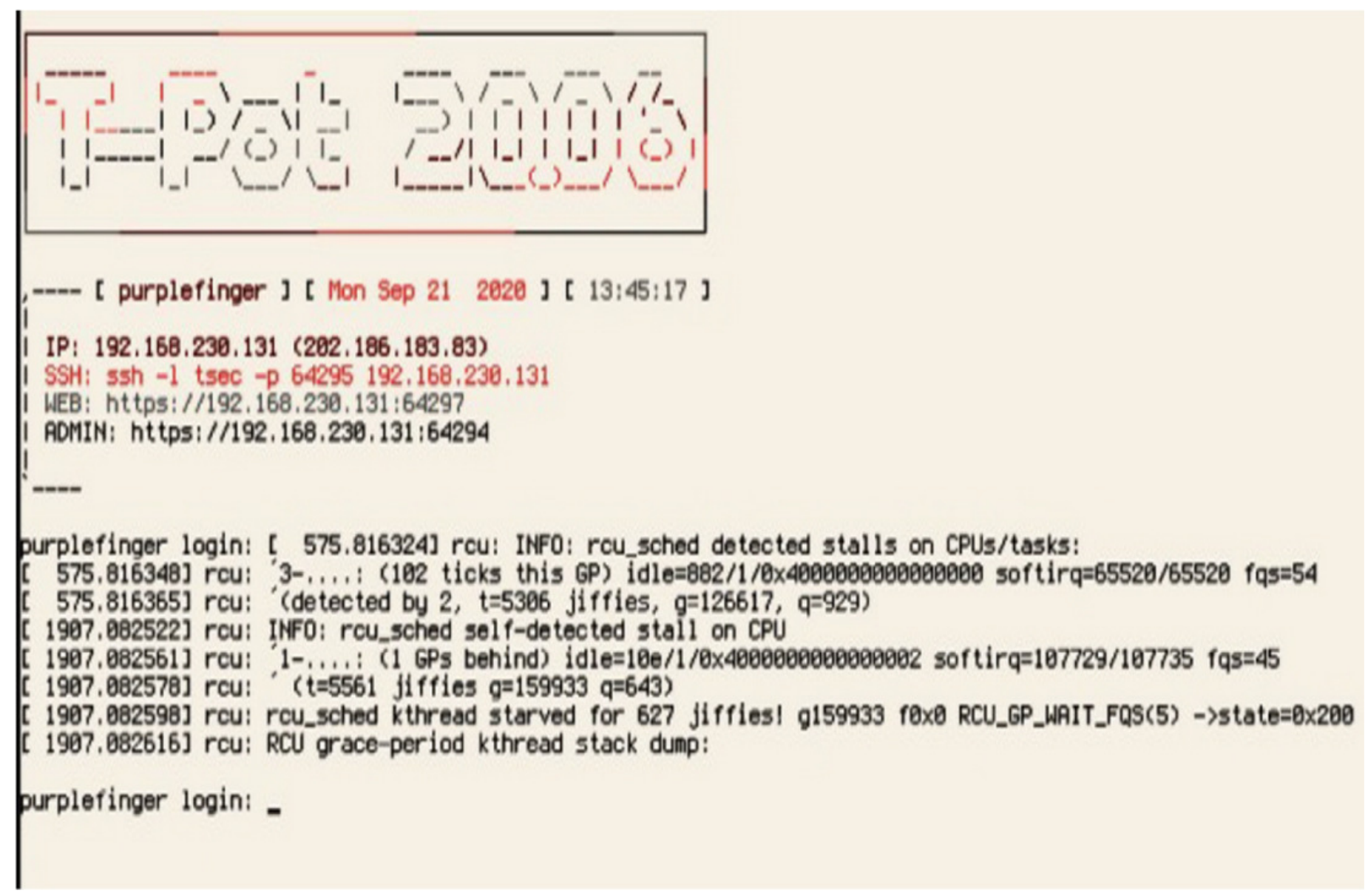




\section{Figure 8}

\section{Kibana dashboard.}

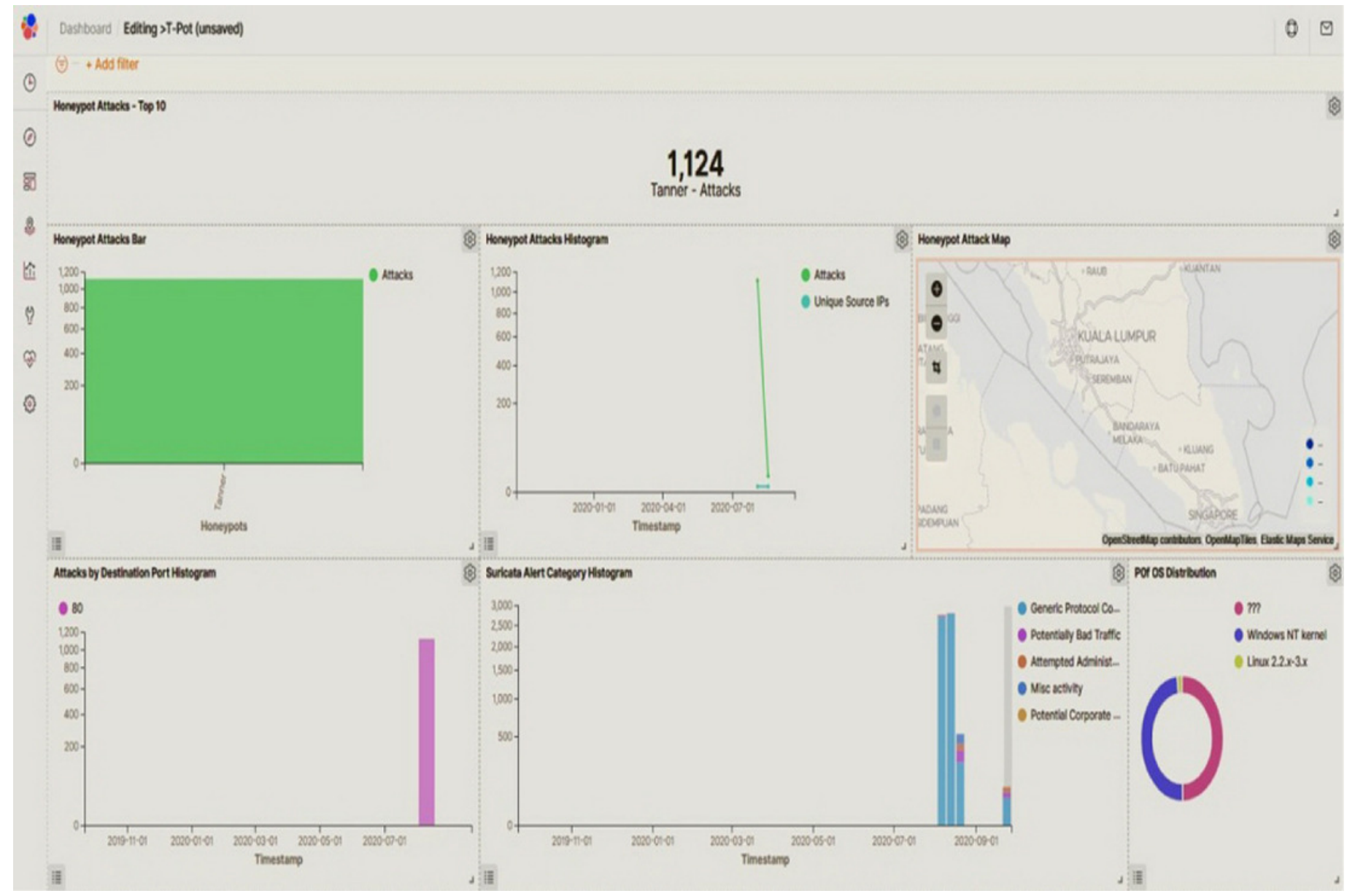




\section{Figure 9}

Process design of honeypot combined machine learning model to detect botnet attacks in smart factory.

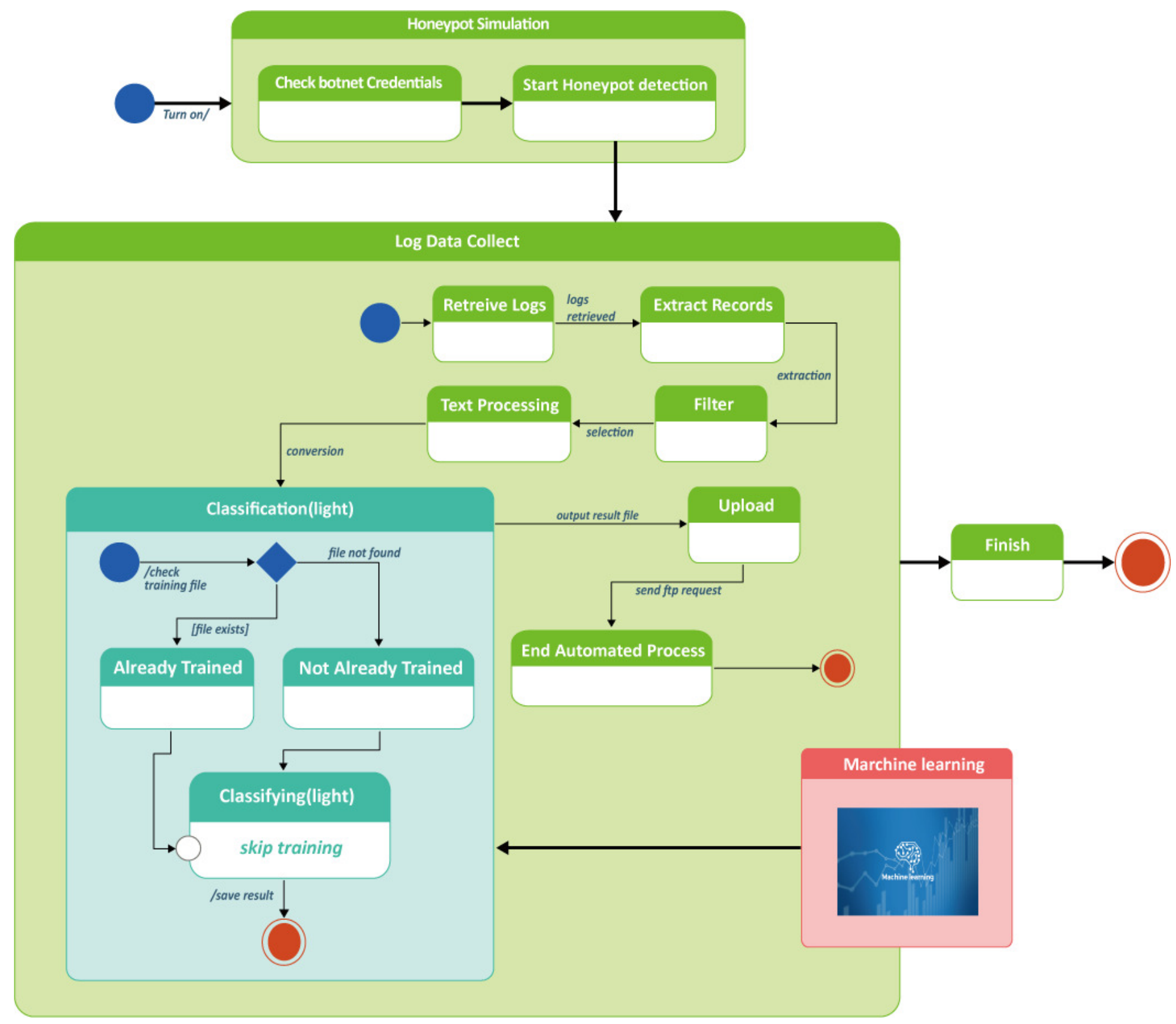




\section{Figure 10}

Correlation of true vs. false positive rates for the 10 best features.

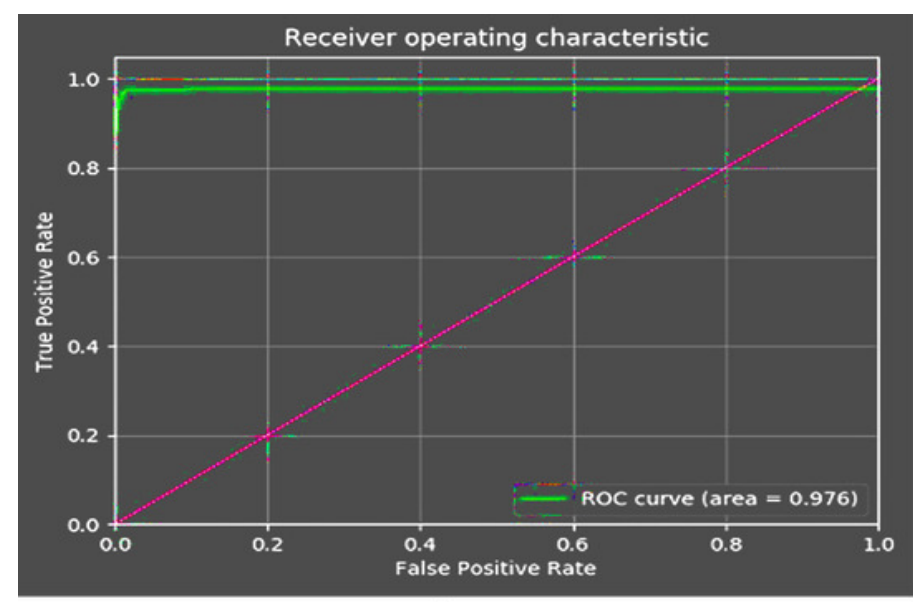

A)

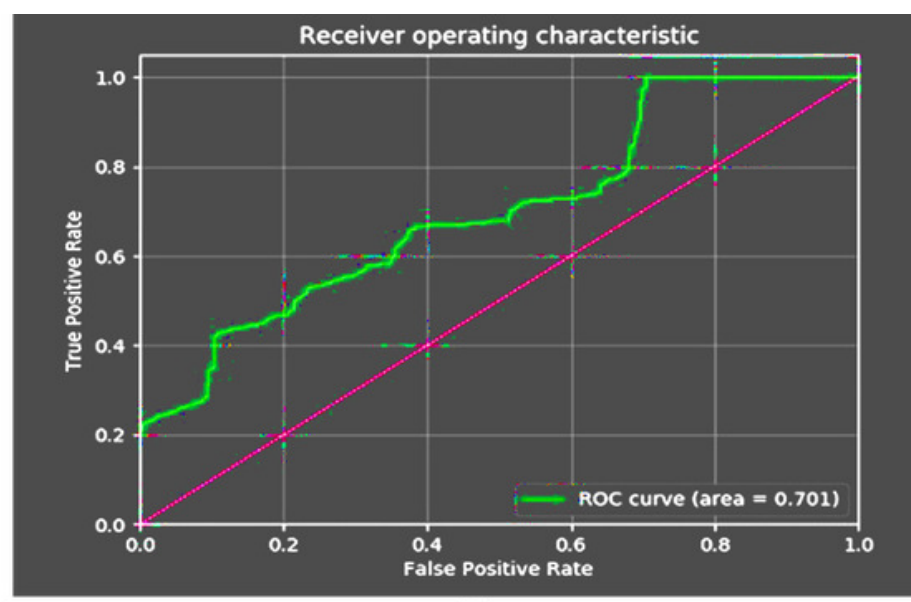

B) 


\section{Figure 11}

Detailed Accuracy by classification.

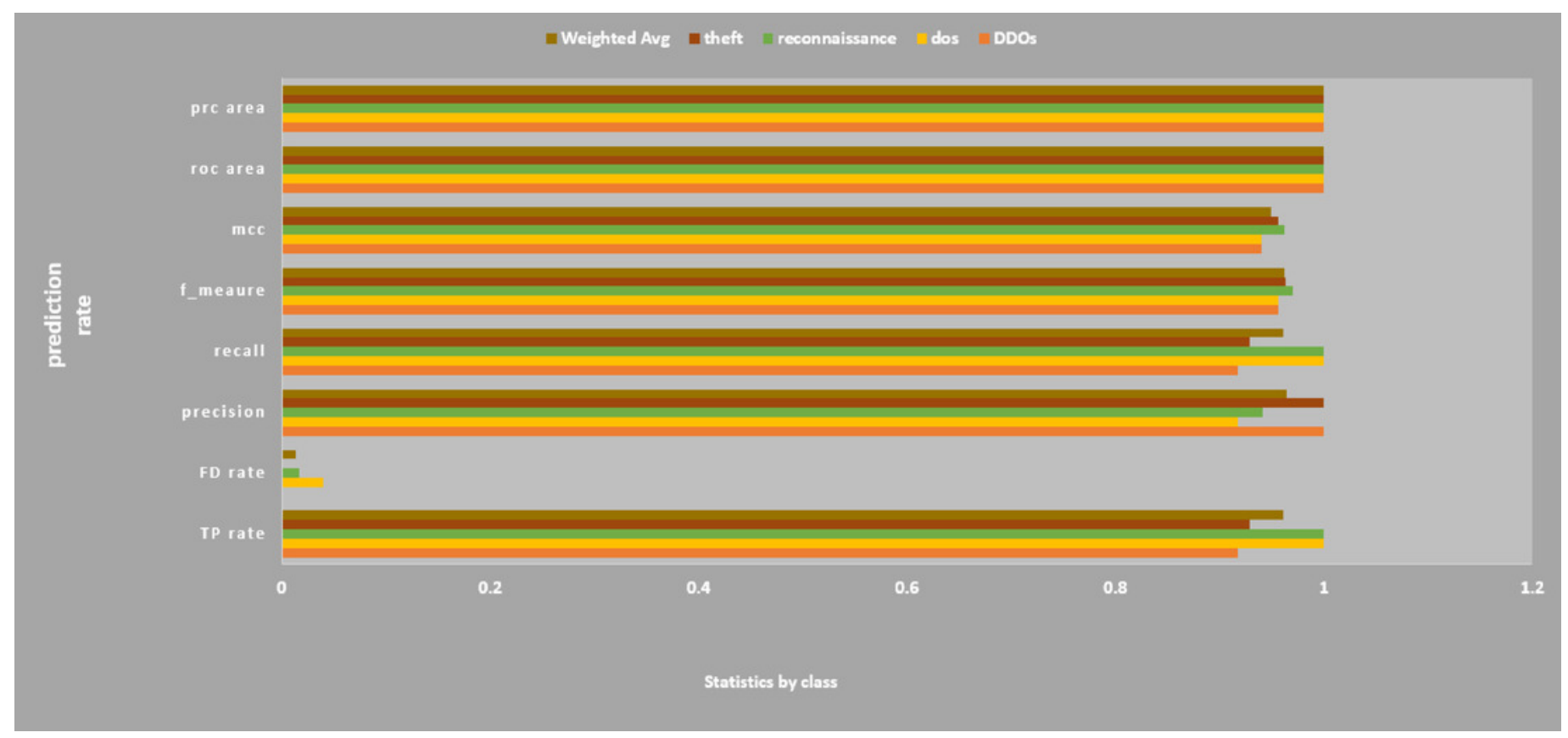




\section{Figure 12}

Correlation of 10 features for Weka-machine learning.

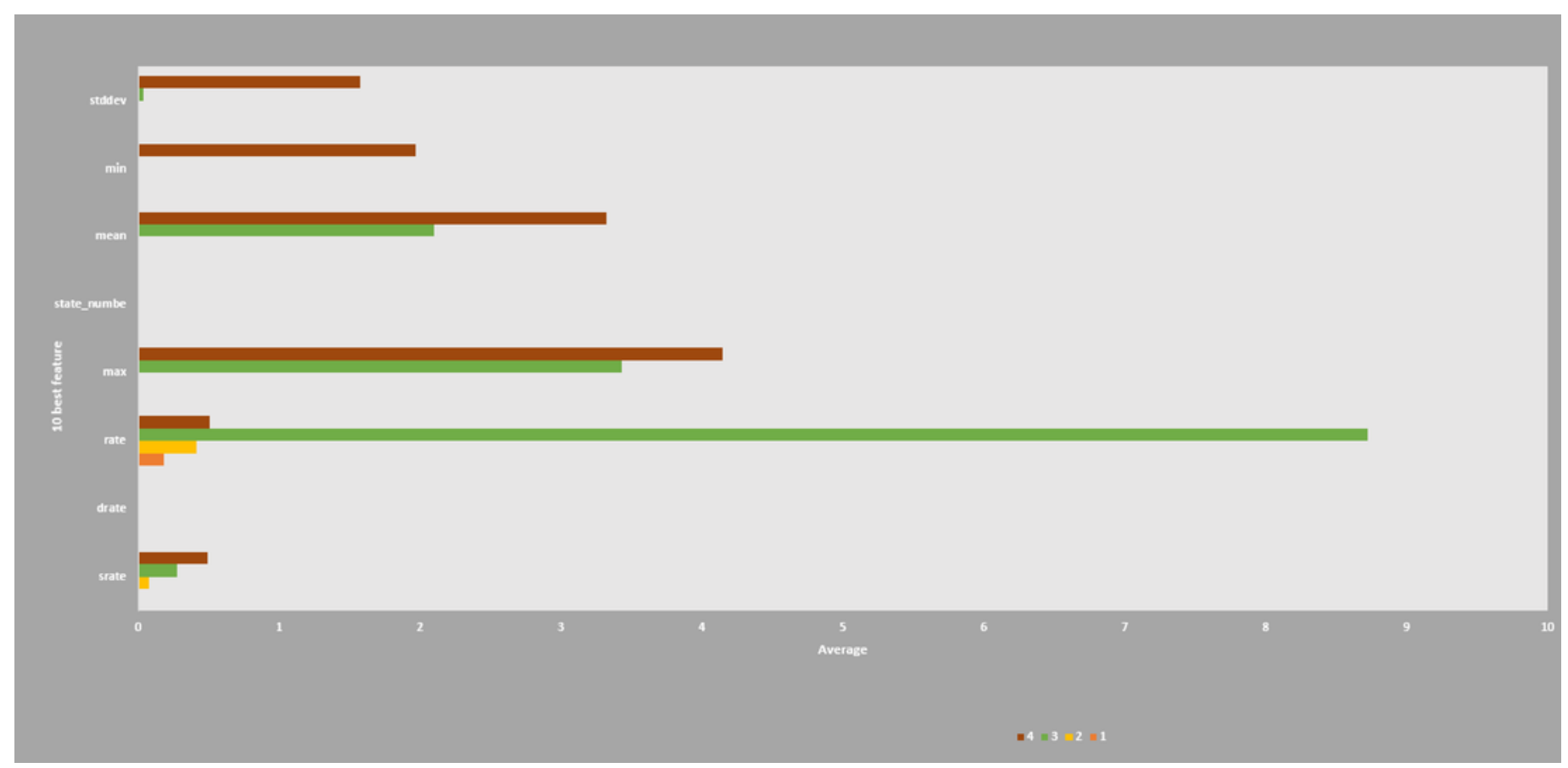




\section{Figure 13}

\section{R-studio results.}
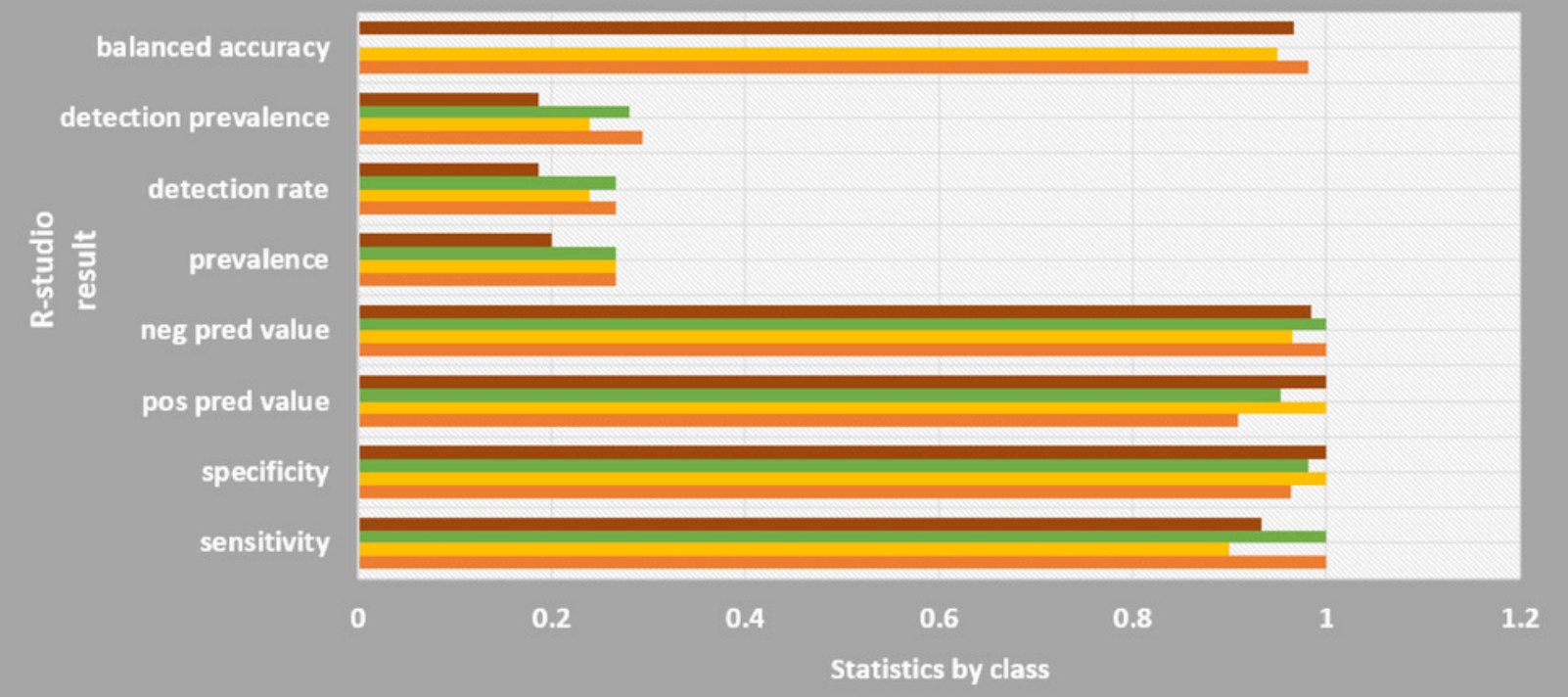

- theft reconnaissance dos Ddos 


\section{Figure 14}

Graphical comparison of random forest in this study with random committee in other study.

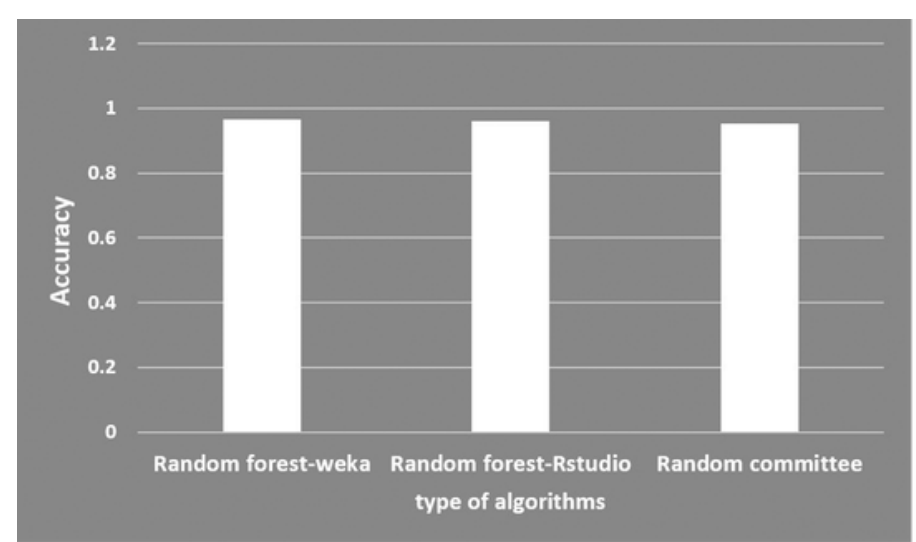

a) Accuracy

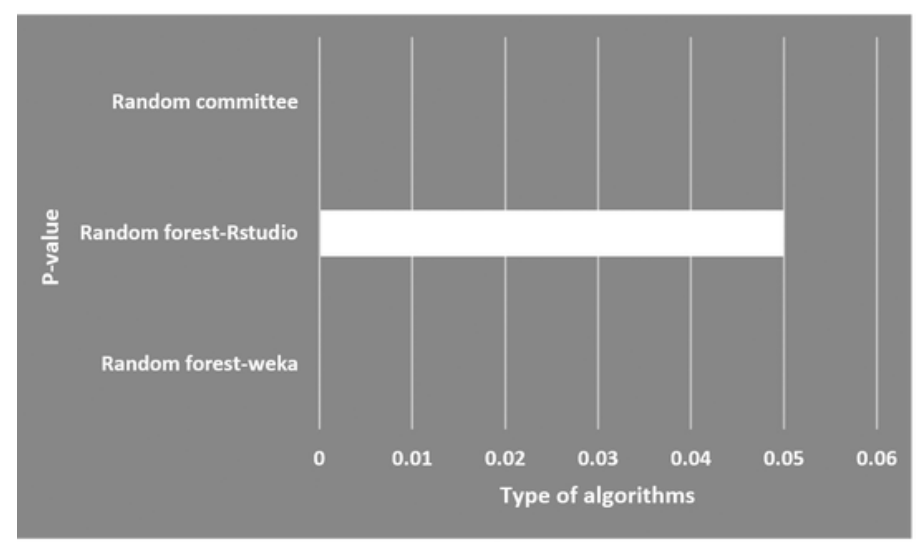

c) P-Value

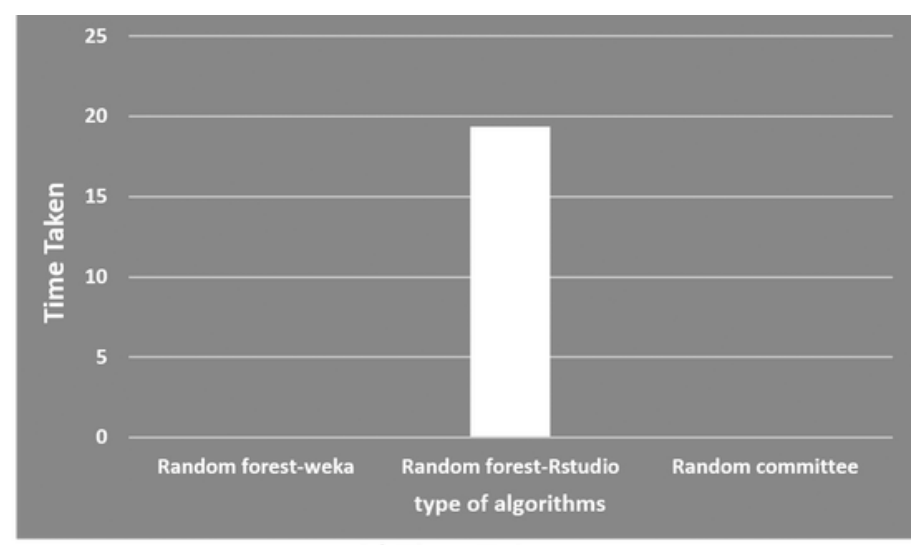

b) Time Taken

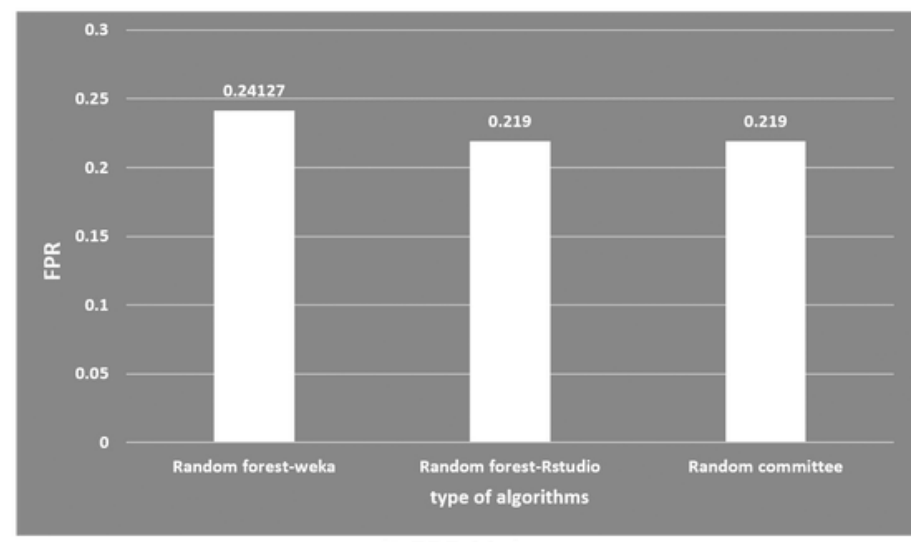

d) FPR Value 